\title{
Quantum Damped Mechanical Oscillator
}

\author{
Akpan N. Ikot, ${ }^{1}$ Louis E. Akpabio, ${ }^{1}$ Ita O. Akpan, ${ }^{2}$ Michael I. Umo, ${ }^{2}$ and Eno E. Ituen ${ }^{1}$ \\ ${ }^{1}$ Department of Physics, University of Uyo, Uyo, Nigeria \\ ${ }^{2}$ Department of Physics, University of Calabar, Calabar, Nigeria
}

Correspondence should be addressed to Akpan N. Ikot, ndemikot2005@yahoo.com

Received 16 October 2009; Revised 6 February 2010; Accepted 8 March 2010

Academic Editor: Ortunato Tito Arecchi

Copyright ( 2010 Akpan N. Ikot et al. This is an open access article distributed under the Creative Commons Attribution License, which permits unrestricted use, distribution, and reproduction in any medium, provided the original work is properly cited.

\begin{abstract}
The exact solutions of the Schrödinger equation for quantum damped oscillator with modified Caldirola-Kanai Hamiltonian are evaluated. We also investigate the cases of under-, over-, and critical damping.
\end{abstract}

\section{Introduction}

Quantum theory has been shown to be the fundamental law of nature and presently is the most correct theory of all microscopic and macroscopic systems [1-3]. Quantum effects usually manifested themselves at the microscopic level. The quantum theory is, however, governed by the Schrödinger equation whereas classical theory is governed by the Hamilton equation, for instance [3]. Dissipation is usually ascribed as having a microscopic nature. There have been attempts to understand dissipation at a more fundamental level [1-26]. The simplest model for dissipation is damped oscillators with one or two degrees of freedom. In the canonical approach, two different Hamiltonian representations have been introduced for these damped oscillators. One representation of the damped system is the Caldirola-kanai (CK) oscillator which is a one-dimensional system with an exponentially increasing mass [1-3, 18-20, 23-26]. Another representation is the Bateman-Feshbach-Tikochinsky (BFT) oscillator, which consists of a damped and an amplified oscillator [3,23-26]. The CK, on one hand, is an open system whose parameters such as mass and frequency are all time dependent $[1,2]$, and, on the other hand, the BFT is a closed system whose total energy is conserved and the dissipated energy from the damped oscillator is transferred to amplified $[3,26]$. Recently, Tarasov has evaluated the quantization and classical distribution for dissipative systems [27, 28]. The aim of this paper is to evaluate the damped harmonic mechanical oscillator. The damping is here considered in the form of Caldirola-Kanai Model [1, 2] and the recently developed model [4]. However, the problem of quantum oscillator with time-varying frequency had been solved [5-12]. The Hamiltonian of this model is usually quadratic in coordinates and momenta operators [4-7]. Our primary goals will be to construct the Lagrangian for this simple damped system and use the constructed Lagrangian to evaluate the equation of motion for the damped Harmonic oscillators and also evaluate the minimum uncertain relation for each damping regime.

\section{Review of Bateman-Feshbach- Tikochinsky Oscillator}

In a genuine dissipative system, the energy of the damped subsystem of the system must be dissipated away and transferred to another subsystem. This invariably means that the damped oscillator is described by a two-dimensional system; one subsystem of which dissipates the energy and the other of which gets amplified by the transferred energy. This kind of model has been suggested long ago by Bateman [23] and later by Morse and Feshbach [25] and Feshbach and Tikochinsky [24]. The equation of motion of the onedimensional damped harmonic oscillator is

$$
m \ddot{q}+\gamma \dot{q}+k q=0,
$$

where the parameters $m, \gamma, k$ are time independent. However, since the system in (1) is dissipative, a straightforward Lagrangian description leading to a consistent canonical quantization is not available [29]. In order to develop 
a canonical formalism, one requires (1) alongside its reversed image counterpart [29]:

$$
m \ddot{y}-\gamma \dot{y}+k y=0 .
$$

so that the composite system is conservative. The BFT oscillator is now described by the Lagrangian of systems in (1) and (2) as follows:

$$
L=m \dot{q} \dot{y}+\frac{\gamma}{2}(q \dot{y}-\dot{q} y)-k q y
$$

where $q$ is the damped harmonic oscillator coordinate and $y$ corresponds to the time-reversed counterpart. The Hamiltonian is given by

$$
H_{\gamma}=\frac{1}{m} P_{q} P_{y}+\frac{\gamma}{2 m}\left(y P_{y}-q P_{q}\right)+m \omega^{2} q y,
$$

where

$$
P_{q}=\left(m \dot{y}-\frac{\gamma q}{2}\right), \quad P_{y}=\left(m \dot{q}-\frac{\gamma q}{2}\right) .
$$

The BFT oscillator is just the sum of two-decoupled oscillator with opposite signs in the limit of zero dissipation $(r=0)$ :

$$
H_{0}=\frac{1}{2 m} P_{\xi}^{2}+\frac{k}{2} \xi^{2}-\frac{1}{2 m} P_{o}^{2}-\frac{k}{2} \eta^{2},
$$

where we have introduced the hyperbolic coordinates $x$ and $y$ as

$$
\xi=\frac{1}{\sqrt{2}}(q+y), \quad \eta=\frac{1}{\sqrt{2}}(q-y)
$$

\section{Caldirola-Kanai and Modified Caldirola-Kanai Oscillators}

The CK oscillator with a variable mass $m(t)=m e^{\gamma t / m}$ has Hamiltonian of the form [1-3]

$$
\hat{H}_{\mathrm{ck}}=\frac{1}{2 m e^{\gamma t / m}} p^{2}+\frac{m \omega^{2} e^{\gamma t / m}}{2} q^{2},
$$

or in the form $[1,2,8]$

$$
\hat{H}_{\mathrm{ck}}=\frac{1}{2} m e^{2 \gamma t} \omega^{2}(t) \hat{q}^{2}+\frac{1}{2 m} e^{-2 \gamma t} \hat{p}^{2},
$$

where $m$ is the mass of the oscillator, $\gamma$ is the damping coefficient, $\hat{q}$ and $\hat{p}$ are the coordinate and momentum operators, and $w(t)$ is time-dependent frequency of the oscillator. The Lagrangians associated with (8) and (9) are given as

$$
\begin{aligned}
& L(q, \dot{q}, t)=\frac{m}{2} e^{\gamma t / m}\left[\dot{q}^{2}+\frac{\gamma}{m} \dot{q}+\omega^{2}(t) q\right], \\
& L(q, \dot{q}, t)=\frac{m}{2} e^{\gamma t}\left[\dot{q}^{2}+2 \gamma \dot{q}+\omega^{2}(t) q\right],
\end{aligned}
$$

respectively. The equations of motion for the classical coordinate $q$ and momentum $p$ of (10) and (11) are of the forms

$$
\begin{aligned}
& \ddot{q}(t)+\frac{\gamma}{m} \dot{q}+\omega^{2}(t) q=0, \\
& \ddot{q}(t)+2 \gamma \dot{q}+\omega^{2}(t) q=0 .
\end{aligned}
$$

We write the modified Caldirola-Kanai model [4]

$$
H_{\mathrm{ck}}=\frac{1}{2 m} e^{-\sin \beta \gamma t} p^{2}+\frac{1}{2} m \omega^{2}(t) e^{\sin \beta \gamma t} q^{2},
$$

where (8)-(9) are obtained from (14) when $\sin \beta \gamma t$ is expanded to first order in increasing power of $\beta \gamma t$ with variable parameter $\beta$ being set to 1 . The Lagrangian of this modified Caldirola-Kanai Oscillator becomes

$$
L(q, \dot{q}, t)=\frac{m}{2} e^{\sin \beta \gamma t}\left[\dot{q}^{2}+\gamma \beta \cos \gamma t \dot{q}+\omega^{2}(t) q\right],
$$

and its equation of motion for the classical coordinate $q$ and momentum $p$ takes the form

$$
\ddot{q}(t)+\beta \gamma \cos (\beta \gamma t) \dot{q}(t)+\omega^{2}(t) q=0 .
$$

The solution of (16) is

$$
q(t)=e^{-\beta \gamma t \cos \beta \gamma t / 2}\left[A e^{i \Omega t}+B e^{-i \Omega t}\right],
$$

where $\Omega(t)=\sqrt{4 \omega^{2}(t)-\beta^{2} \gamma^{2} \cos ^{2} \beta \gamma t}$ and approximating $\cos ^{2} \beta \gamma t \simeq 1$ for small damping yields

$$
\Omega(t)=\sqrt{4 \omega^{2}(t)-\beta^{2} \gamma^{2}} .
$$

Substituting (18) into (17) results in

$$
\begin{aligned}
q(t)= & e^{-\beta \gamma t \cos \beta \gamma t / 2} \\
& \times\left[A e^{i \omega t \sqrt{1-\left(\beta^{2} \gamma^{2} / 4 \omega^{2}\right)}}+B e^{-i \omega t \sqrt{1-\left(\beta^{2} \gamma^{2} / 4 \omega^{2}\right)}}\right] .
\end{aligned}
$$

We summarized the general solution of (16) for the overdamped (OD), critically damped (CD), and under-damped (UD) as

$$
\begin{gathered}
q(t)=e^{-\beta \gamma t \cos \beta \gamma t / 2}[A \cosh \Omega t+B \sinh \Omega t], \\
q(t)=e^{-(\beta \gamma t / 2) \cos \beta \gamma t}[A+B t], \\
q(t)=e^{-\beta \gamma t \cos \beta \gamma t / 2}[A \cos \Omega t+B \sin \Omega t],
\end{gathered}
$$

respectively.

\section{Investigation of the Under-Damped (UD), Over-Damped (OD), and Critical Damped (CD) Oscillators}

4.1. The Under-Damped Oscillator. We consider the quantum damped oscillator with time-dependent varying frequency given by (17). Subjecting (17) to continuity conditions [8], we obtain the arbitrary constants $A$ and $B$ as

$$
\begin{aligned}
& A_{k}=\left(1-\frac{i \gamma}{2 \Omega}\right), \\
& B_{k}=\frac{i \gamma}{2 \Omega},
\end{aligned}
$$


with $k=(0,1)$ corresponding to delta kick, and the classical trajectory becomes

$$
q(t)=e^{-(\beta \gamma t / 2) \cos \beta \gamma t} \times\left[\left(1-\frac{i \gamma}{2 \Omega}\right) \cos \Omega t+\frac{i \gamma}{2 \Omega} \sin \Omega t\right] .
$$

The wave functions of (8)-(9) and (14) are determined by different methods [13-15], and for the latest review see [16]. An invariant operator for the general time-dependent oscillator whose eigenfunction is an exact quantum state up to a time-dependent phase factor had been introduced by Lewis and Riesenfeld [17]. We introduce a pair of operators first order in position and momentum $[3,8,18-20]$ as follows:

$$
\begin{aligned}
\hat{a}(t) & =i\left[\varepsilon^{*}(t) \hat{p}-\dot{\varepsilon}^{*}(t) \hat{q}\right], \\
\hat{a}^{t}(t) & =-i[\varepsilon(t) \hat{p}-\dot{\varepsilon}(t) \hat{q}],
\end{aligned}
$$

and they are required to satisfy the quantum Liouville-von Neumann equations defined as

$$
\begin{aligned}
i \hbar \frac{\partial}{\partial t} \hat{a}(t)+\left[\hat{a}(t), \hat{H}_{\mathrm{ck}}(t)\right] & =0, \\
i \hbar \frac{\partial}{\partial t} \hat{a}^{+}(t)+\left[\hat{a}^{+}(t), \hat{H}_{\mathrm{ck}}(t)\right] & =0,
\end{aligned}
$$

where $\varepsilon(t)$ in (23) must satisfy the classical damped equation of (16).

The operator in (24) and its Hermitian conjugate satisfy at any time $t$ the boson commutation relation [8], and $\varepsilon(t)$ must also satisfy the Wronskian condition

$$
e^{(d / d \beta) \sin \beta \gamma t}\left[\dot{\mathcal{\varepsilon}}^{*}(t) \mathcal{\varepsilon}(t)-\dot{\varepsilon}(t) \mathcal{E}^{*}(t)\right]=i,
$$

where $\Omega^{2}(0)=4 \omega^{2}(0)-\gamma^{2}$. The number operator defined by

$$
\hat{N}(t)=\hat{a}^{+}(t) \hat{a}(t)
$$

also satisfies (25) [3] such that each number state

$$
\widehat{N}(t)|n, t\rangle=n|n, t\rangle
$$

is also an exact quantum state of the time-dependent Schrödinger equation

$$
i \hbar \frac{\partial}{\partial t} \Psi(x, t)=\hat{H}_{\mathrm{ck}}(t) \Psi(x, t)
$$

where $\hat{H}_{\mathrm{ck}}(t)$ is the modified Caldirola-Kanai Hamiltonian of (14). The wave function that satisfies (14) can be written as [22]

$$
\begin{aligned}
\psi_{\alpha}(q, t)= & \left(\frac{\pi \varepsilon^{2} \hbar^{2}}{m \Omega(0)}\right)^{-1 / 4} \\
& \times \exp \left[\frac{i \varepsilon \exp (\sin \beta \gamma t) m \Omega(0)}{2 \varepsilon(t) \Omega(0)} q^{2}\right. \\
& +\frac{\sqrt{2} \alpha}{\varepsilon(t)}\left(\frac{m \Omega(0)}{\hbar}\right)^{1 / 2} q \\
& \left.-\frac{\varepsilon^{*}(t) \alpha^{2}}{2 \varepsilon(t)}-\frac{|\alpha|^{2}}{2}\right], \\
\psi_{n}(q, t)= & \left(\frac{\pi \varepsilon^{2}(t) \hbar}{m \Omega(0)}\right)^{-1 / 4}\left(\frac{\varepsilon^{*}(t)}{2 \varepsilon(t)}\right)^{n / 2} \frac{1}{\sqrt{n !}} \\
& \times \exp \left[\frac{i \dot{\varepsilon}(t) m \Omega(0) e^{\sin \beta \gamma t}}{2 \hbar \varepsilon(t)} q^{2}+\right] H_{n}\left(\frac{q}{\sqrt{\varepsilon \varepsilon^{*}}}\right),
\end{aligned}
$$

where $\alpha$ in (31) is a complex number and the wave function in co-ordinate representations of (31)-(32) are Gaussian packets with time-dependent coefficients in quadratic form under the exponential function [8].

We obtain the quantum dispersion coordinate in the form

$$
\begin{aligned}
\left\langle\hat{q}^{2}\right\rangle & =\hbar^{2} \varepsilon^{*}(t) \varepsilon(t) \\
& =\frac{\hbar e^{-\beta \gamma t \cos \beta \gamma t}}{2 m \Omega}\left[1+\frac{\beta^{2} \gamma^{2}}{\Omega^{2}} \sin ^{2} \Omega t+\frac{\beta \gamma}{\Omega} \sin 2 \Omega t\right],
\end{aligned}
$$

and the uncertainty in momentum is

$$
\begin{aligned}
\left\langle\hat{p}^{2}\right\rangle= & \hbar^{2} m^{\prime 2}(t) \dot{\varepsilon}^{*}(t) \dot{\varepsilon}(t) \\
= & \frac{\hbar m \omega^{2}}{2 \Omega} e^{(2-\beta) \gamma t \cos \beta \gamma t h} \\
\times & {\left[1+\frac{\beta \gamma^{2}}{\Omega^{2}} \sin ^{2} \Omega t+\left(\frac{\sigma(t)}{\Omega}\right)^{2}\right.} \\
& \times\left(\frac{\beta \gamma}{2 \Omega} \cos 2 \Omega t-\frac{\beta \gamma}{\Omega} \sin 2 \Omega t-\frac{1}{2}\left(\frac{\beta \gamma}{\Omega}\right)^{2}-1\right) \\
& \left.+\frac{\beta \gamma \sigma(t)}{\Omega^{2}}\left(\frac{\beta \gamma}{\Omega} \sin 2 \Omega t-2 \cos 2 \Omega t\right)+\frac{\beta \gamma}{\Omega} \sin 2 \Omega t\right],
\end{aligned}
$$

where the quantity $\sigma(t)$ is given as

$$
\sigma(t)=\frac{\beta \gamma}{2}(\cos \beta \gamma t+\beta \gamma t \sin \beta \gamma t),
$$

and the reduced mass $m^{\prime}(t)$ of the modified oscillator is defined as [4]

$$
m^{\prime}(t)=m e^{(d / d \beta)(\sin \beta \gamma t)} .
$$




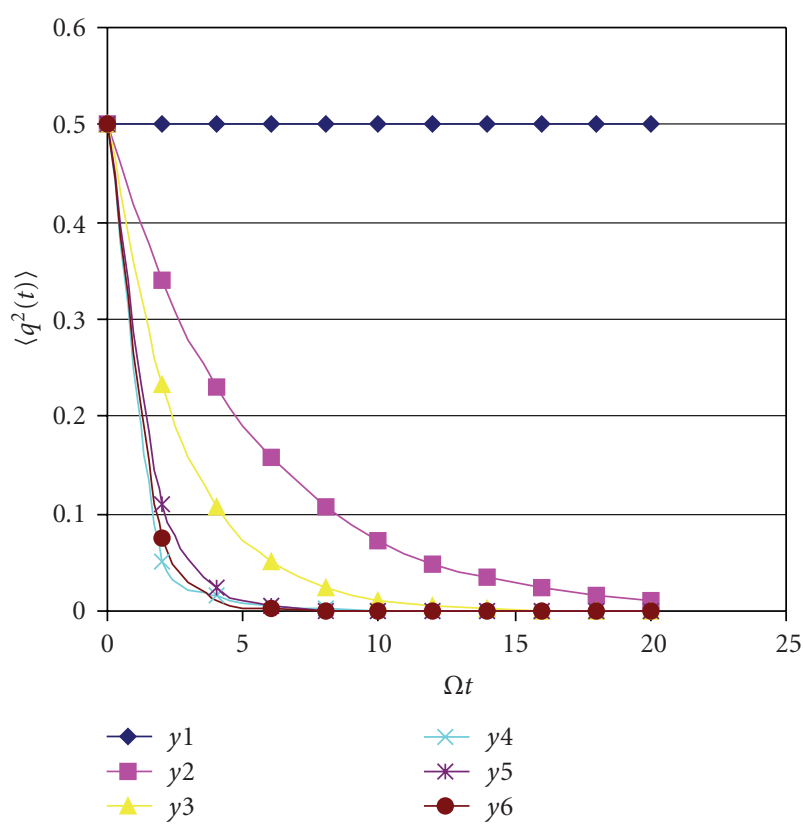

FIgURE 1: The uncertainties in position for the under-damped oscillator as a function of $\Omega t$ are governed by the modified Caldirola-Kanai Hamiltonian with dissipation coefficients $\gamma=0$, $0.2 \Omega, 0.4 \Omega, 0.6 \Omega, 0.8 \Omega$, and $\Omega$, respectively.

These results show that the spreading of the wave packet is suppressed by the appearance of dissipation [21]. However, the generalized uncertainty relation has the value

$$
\Delta q \Delta p=\frac{\hbar \omega}{2 \Omega} e^{(1-\beta) \gamma t \cos \beta \gamma t}[1+\Lambda(t)]^{1 / 2},
$$

where

$\Lambda(t)$

$$
\begin{aligned}
=(1+ & \left.\frac{\beta^{2} \gamma^{2}}{\Omega^{2}} \sin ^{2} \Omega t+\frac{\beta \gamma}{\Omega} \sin 2 \Omega t\right) \\
\times & \left\{1+\frac{\beta^{2} \gamma^{2}}{\Omega^{2}} \sin ^{2} \Omega t+\left(\frac{\sigma(t)}{\Omega}\right)^{2}\right. \\
& \times\left[\frac{1}{2}\left(\frac{\beta \gamma}{\Omega}\right)^{2} \cos 2 \Omega t-\frac{\beta \gamma}{\Omega} \sin 2 \Omega t-\frac{1}{2}\left(\frac{\beta \gamma}{\Omega}\right)^{2}-1\right] \\
& \left.+\frac{\beta \gamma \sigma(t)}{\Omega^{2}}\left[\frac{\beta \gamma}{\Omega} \sin 2 \Omega t-2 \cos 2 \Omega t\right]+\frac{\gamma \beta}{\Omega} \sin 2 \Omega t\right\} .
\end{aligned}
$$

Equation (37a)-(37b) is a generalized uncertainty relation and it satisfies the Heisenberg Uncertainty relation when the variable parameter $\beta$ is set to unity. Figure 1 shows the uncertainty in coordinate for $\gamma=0,0.2 \Omega$, $0.4 \Omega, 0.6 \Omega, 0.8 \Omega$, and $\Omega$. The products of (32) and (33) give a generalized Heisenberg relation which reduces to the exact when the variable parameter $\beta$ is set to unity and the damping coefficient $\gamma$ is set to zero.
4.2. The Over-Damped Oscillator. The Over-damping occurs when the damping factor $\gamma>\omega$. When this happens, the solution to the classical trajectory takes the form and imposing the boundary conditions [8] leads to

$$
q(t)=e^{-\beta \gamma t \cos \beta \gamma t / 2}\left[\cosh \Omega t+\left(i+\frac{\beta \gamma}{\Omega}\right) \sinh \Omega t\right] .
$$

We obtain the uncertainty in the coordinate as

$$
\begin{aligned}
\left\langle\hat{q}^{2}\right\rangle= & \frac{\hbar e^{-\beta \gamma t \cos \beta \gamma t / 2}}{2 m \Omega} \\
& \times\left[\cosh 2 \Omega t+\frac{\beta \gamma}{\Omega} \sinh 2 \Omega t+\left(\frac{\beta \gamma}{\Omega}\right)^{2} \sinh ^{2} \Omega t\right] .
\end{aligned}
$$

Similarly, the dispersion of momentum takes the form

$$
\begin{aligned}
\left\langle p^{2}\right\rangle= & \frac{\hbar m \omega^{2}}{2 \Omega} e^{(2-\beta) \gamma t \cos \beta \gamma t} \\
\times & {\left[\cosh 2 \Omega t+\left(\frac{\sigma(t)}{\Omega}\right)^{2} \cosh 2 \Omega t\right.} \\
& \left.-2\left(\frac{\sigma(t)}{\Omega}\right) \sinh 2 \Omega\right] \\
- & \left(\frac{\gamma}{\Omega}\right)^{2}\left[\cosh 2 \Omega t+\left(\frac{\sigma(t)}{\Omega}\right) \cosh 2 \Omega t\right. \\
& \left.+2\left(\frac{\sigma(t)}{\Omega}\right) \sinh 2 \Omega t\right] \\
- & \left(\frac{r}{\Omega}\right)^{2}\left[\sinh 2 \Omega t+\left(\frac{\sigma(t)}{\Omega}\right)^{2} \sinh 2 \Omega t\right. \\
& \left.+2\left(\frac{\sigma(t)}{\Omega}\right) \cosh 2 \Omega t\right] .
\end{aligned}
$$

However, since $\cosh 2 \Omega t \geq 1$, in (39) and (40), then the dispersion cannot be less than $\hbar e^{-\beta \gamma t \cos \beta \gamma t} / 2 m \Omega$ and $\left(\hbar m \omega^{2} / 2 \Omega\right) e^{-\beta \gamma t \cos \beta \gamma t}$ in both equations, respectively. Figure 2 shows the uncertainties in the coordinate for the Overdamped Oscillator for various damping factors of $\gamma=0$, $0.2 \Omega, 0.4 \Omega, 0.6 \Omega, 0.8 \Omega$, and $\Omega$, respectively.

4.3. The Critical Damped Oscillator. The equation for the critical damped oscillator is of the form, when $\omega=0$,

$$
q(t)=A_{k}+B_{k} e^{-\beta \gamma t \cos \beta \gamma t / 2} .
$$

Subjecting (41) to continuity condition [8],

$$
q(t)=1+\frac{i}{2}\left(1-e^{-\beta \gamma t \cos \beta \gamma t / 2}\right) .
$$

The uncertainty in the coordinate in space is given by

$$
\left\langle q^{2}(t)\right\rangle=\hbar^{2}\left[1+\frac{1}{4}\left(1-e^{-\beta \gamma t \cos \beta \gamma t / 2}\right)^{2}\right],
$$




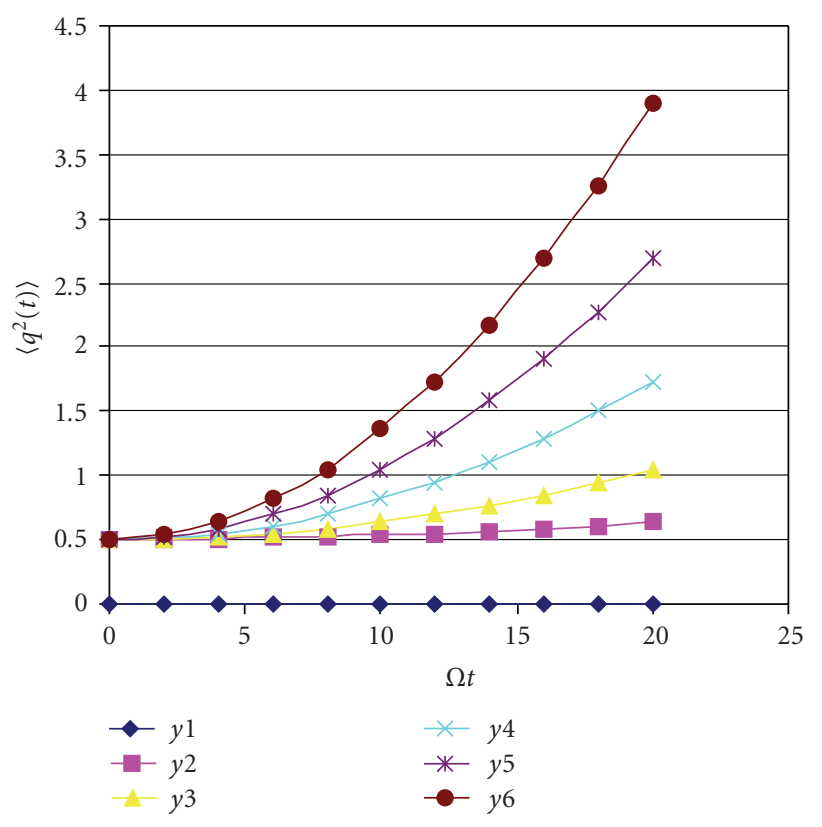

FIgURE 2: The uncertainties in position for the over-damped oscillator as a function of $\Omega t$ is governed by the modified CaldirolaKanai Hamiltonian with dissipation coefficients $\gamma=0,0.2 \Omega, 0.4 \Omega$, $0.6 \Omega, 0.8 \Omega$, and $\Omega$, respectively.

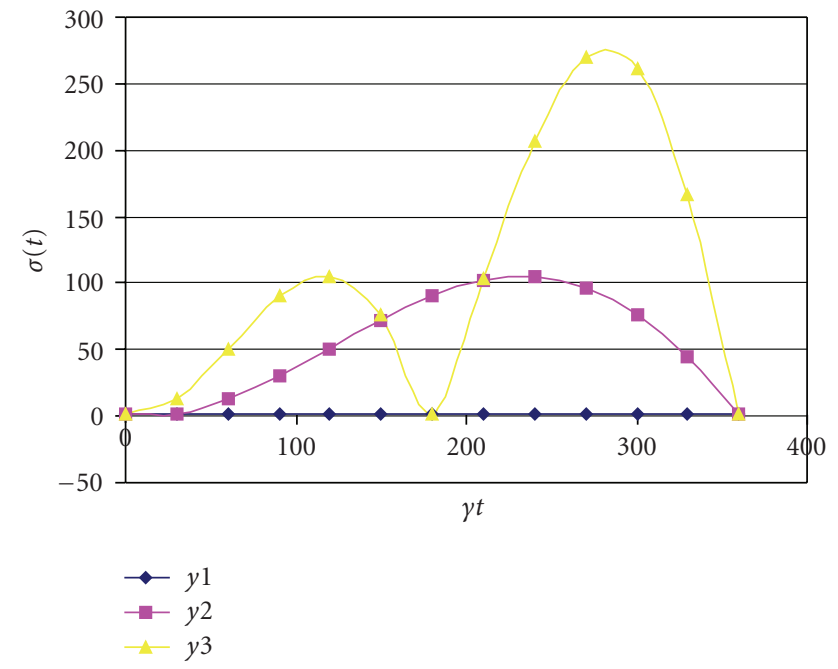

FIGURE 3: Variation of $\sigma(t)$ with $\gamma t$ for the critical damped oscillator with various damping factors of $0,0.5 \Omega$, and $\Omega$.

and dispersion in the momentum counterpart is

$$
\left\langle p^{2}(t)\right\rangle=\frac{\hbar}{4} e^{(2-\beta) \gamma t \cos \beta \gamma t} \sigma(t)^{2}(t) .
$$

We observed in (43) that $e^{-\beta \gamma t \cos \beta \gamma t / 2}<1$, so that the product of dispersion becomes

$$
\begin{array}{r}
\langle\Delta p \Delta q\rangle=\frac{\hbar \beta \gamma}{2}\left[\cos ^{2} \beta \gamma t-2 \beta \gamma t \sin 2 \beta \gamma t\right. \\
\left.+\gamma^{2}+\beta^{2} \gamma^{2} t^{2} \sin ^{2} \beta \gamma t\right]^{1 / 2} .
\end{array}
$$

When the variable parameter is set to unity, Figure 3 shows the variation of $\sigma^{2}(t)$ in (44) for various damping factors.

\section{Conclusion}

We have evaluated within the frame of Caldirola-Kanai model the damped harmonic oscillator for different damping regimes. Here, we obtain the modified Caldirola-Kanai Hamiltonian and show that the undamped regime $\gamma<\omega$ satisfied the uncertainty relation with the chosen variable parameter $\beta$ being set to unity. In the region of strong and critical damping, Heisenberg uncertainty relation is violated even when this variable parameter is set to unity.

\section{Acknowledgments}

This work was supported by the Imienyong Nandy and Leabio Research Foundation under Grant no. INL-743-214.

\section{References}

[1] P. Caldirola, "Forze non conservative nella meccanica quantistica," Nuovo Cimento, vol. 18, no. 9, pp. 393-400, 1941.

[2] E. Kanai, "On the quantization of the dissipative systems," Progress of Theoretical Physics, vol. 3, no. 4, pp. 440-442, 1948.

[3] S. P. Kim, A. E. Santana, and F. C. Khanna, "Decoherence of quantum damped oscillators," Journal of the Korean Physical Society, vol. 43, no. 4 I, pp. 452-460, 2003.

[4] A. N. Ikot, E. E. Ituen, I. E. Essien, and L. E. Akpabio, "Path integral evaluation of a time-dependent oscillator in an external field," Turkish Journal of Physics, vol. 32, no. 6, pp. 305-313, 2008.

[5] V. V. Dodonor, I. A. Malkim, and V. I. Man'ko, "Integrals of the motion, green functions, and coherent states of dynamical systems," International Journal of Theoretical Physics, vol. 14, no. 1, pp. 37-54, 1975.

[6] V. V. Dodonov, O.V. Man'ko, and V.T. Man’ko, “Timedependent oscillator with Kronig-Penney excitation," Physics Letters A, vol. 175, no. 1, pp. 1-4, 1993.

[7] Y. S. Kim and V. I. Man'ko, "Time-dependent mode coupling and generation of two-mode squeezed states," Physics Letters A, vol. 157, no. 4-5, pp. 226-228, 1991.

[8] O. V. Man'ko and L. Yeh, "Correlated squeezed states of two coupled oscillators with delta-kicked frequencies," Physics Letters A, vol. 189, no. 4, pp. 268-276, 1994.

[9] T. Kiss, P. Adam, and J. Janszky, "Time-evolution of a harmonic oscillator: jumps between two frequencies," Physics Letters A, vol. 192, no. 5-6, pp. 311-315, 1994.

[10] C. I. Um, K. H. Yeon, and W. H. Kahng, "The quantum damped driven harmonic oscillator," Journal of Physics A, vol. 20, no. 3, p. 611, 1987.

[11] K. H. Yeon, C. I. Um, and T. F. George, "Coherent states for the damped harmonic oscillator," Physical Review A, vol. 36, no. 11, pp. 5287-5291, 1987.

[12] K. H. Yeon, C. T. Um, W. H. Kahng, and T. F. George, "Propagators for driven coupled harmonic oscillators," Physical Review A, vol. 38, no. 12, pp. 6224-6230, 1988.

[13] V. V. Dodonov and V. I. Man'Ko, "Coherent states and the resonance of a quantum damped oscillator," Physical Review A, vol. 20, no. 2, pp. 550-560, 1979. 
[14] J. M. Cervero and J. Villarroel, "On the quantum theory of the damped harmonic oscillator," Journal of Physics A, vol. 17, no. 15, pp. 2963-2971, 1984.

[15] R. K. Colegrave and M. Sebawe Abdalla, "Harmonic oscillator with exponentially decaying mass," Journal of Physics A, vol. 14, no. 9, pp. 2269-2280, 1981.

[16] C.-I. Um, K.-H. Yeon, and T. F. George, "The quantum damped harmonic oscillator," Physics Report, vol. 362, no. 23, pp. 63-192, 2002.

[17] H. R. Lewis Jr. and W. B. Riesenfeld, "An exact quantum theory of the time-dependent harmonic oscillator and of a charged particle in a time-dependent electromagnetic field," Journal of Mathematical Physics, vol. 10, no. 8, pp. 1458-1473, 1969.

[18] S. P. Kim and D. N. Page, "Classical and quantum action-phase variables for time-dependent oscillators," Physical Review A, vol. 64, no. 1, pp. 121041-121048, 2001.

[19] J. K. Kim and S. P. Kim, "One-parameter squeezed Gaussian states of a time-dependent harmonic oscillator and the selection rule for vacuum states," Journal of Physics A, vol. 32, no. 14 , pp. 2711-2718, 1999.

[20] S. P. Kim and C. H. Lee, "Nonequilibrium quantum dynamics of second order phase transitions," Physical Review D, vol. 62, no. 12, Article ID 125020, pp. 1-28, 2000.

[21] M. Huang and M. Wu, "The Caldirora-Kanai model and its equivalent theories for a damped oscillator," Chinese Journal of Physics, vol. 36, no. 4, pp. 566-587, 1998.

[22] V. I. Man'ko and S. S. Safonov, "The damped quantum oscillator and a classical representation of quantum mechanics," Theoretical and Mathematical Physics, vol. 112, no. 3, pp. 1172-1181, 1997.

[23] H. Bateman, "On dissipative systems and related variational principles," Physical Review Letters, vol. 38, no. 4, pp. 815-819, 1931.

[24] H. Feshbach and Y. Tikochinsky, "Quantization of the damped harmonic oscillator," Transactions of the New York Academy of Sciences, vol. 38 II, no. 1, pp. 44-53, 1977.

[25] P. M. Morse and H. Feshbach, Methods of Theoretical Physics, vol. 1, McGraw-Hill, New York, NY, USA, 1953.

[26] E. Celleghini, M. Rasetti, and G. Vitiello, "Quantum dissipation," Annals of Physics, vol. 215, no. 1, pp. 156-170, 1992.

[27] V. E. Tarasov, "Quantization of non-Hamiltonian and dissipative systems," Physics Letters A, vol. 288, no. 3-4, pp. 173-182, 2001.

[28] V. E. Tarasov, "Classical canonical distribution for dissipative systems," Modern Physics Letters B, vol. 17, no. 23, pp. 12191226, 2003.

[29] R. Banerjee and P. Mukhejee, "A canonical approach to the quantization of the damped harmonic oscillator," Journal of Physics A: Mathematical and General, vol. 35, no. 27, pp. 55915598, 2002. 

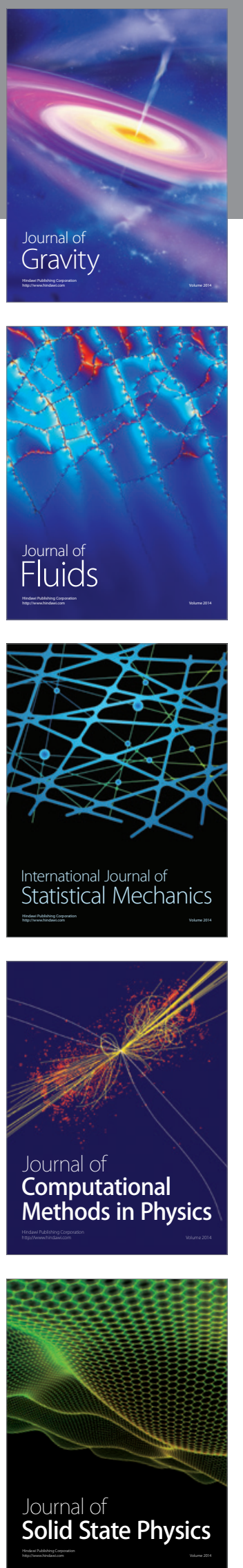

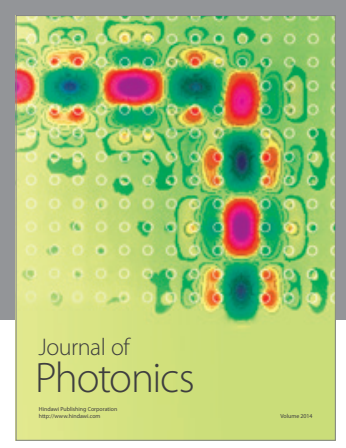

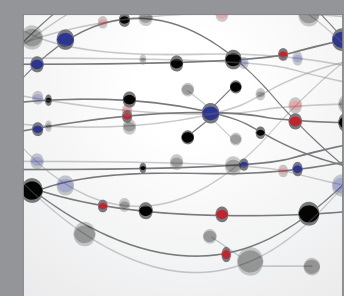

The Scientific World Journal
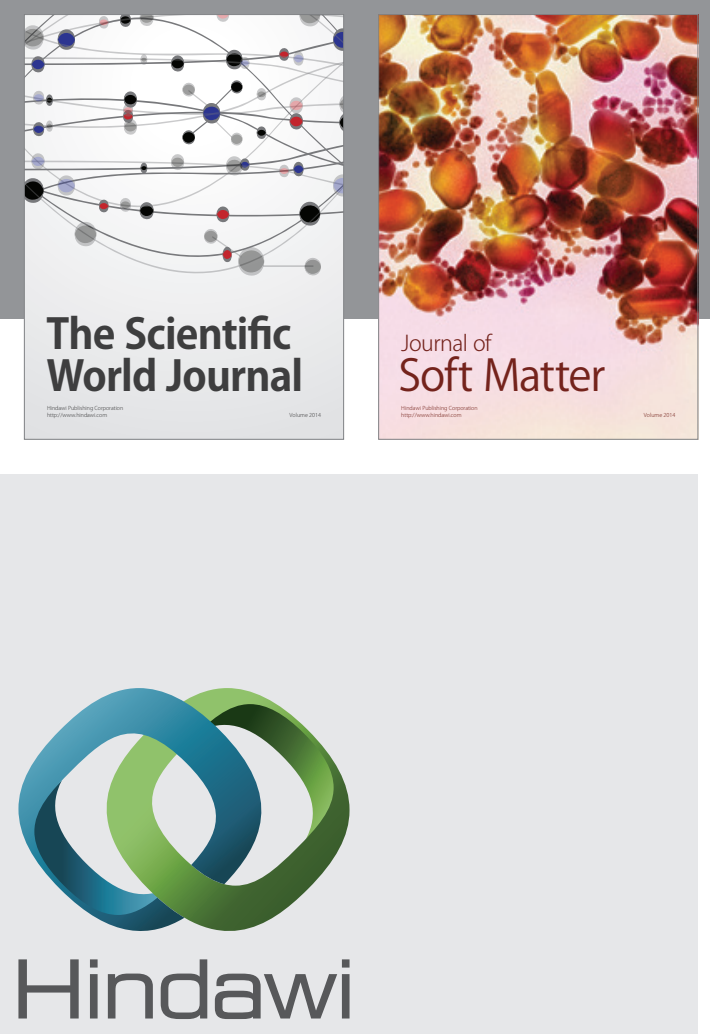

Submit your manuscripts at

http://www.hindawi.com
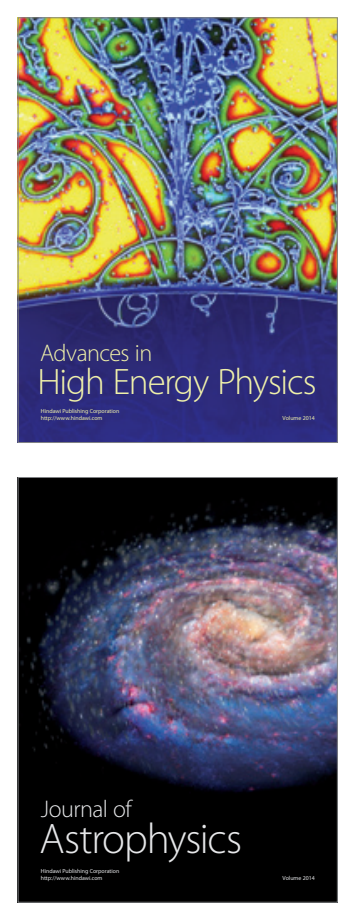
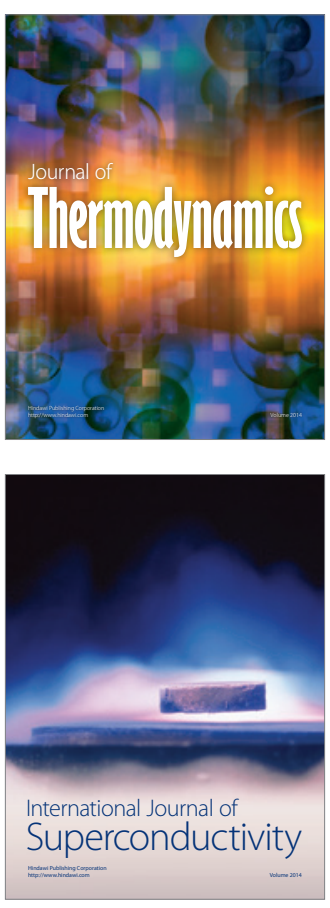
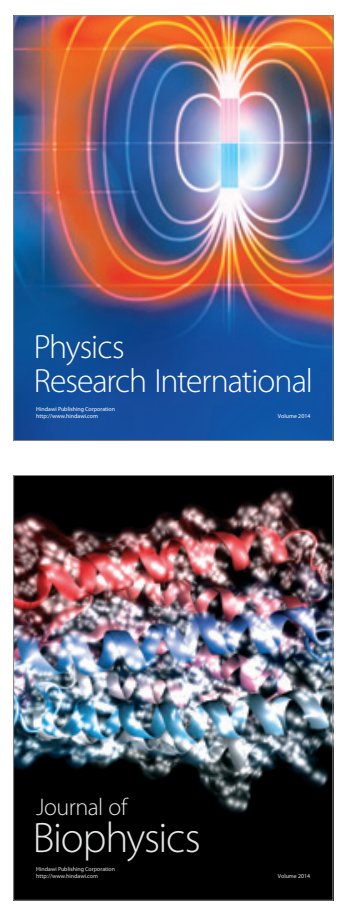
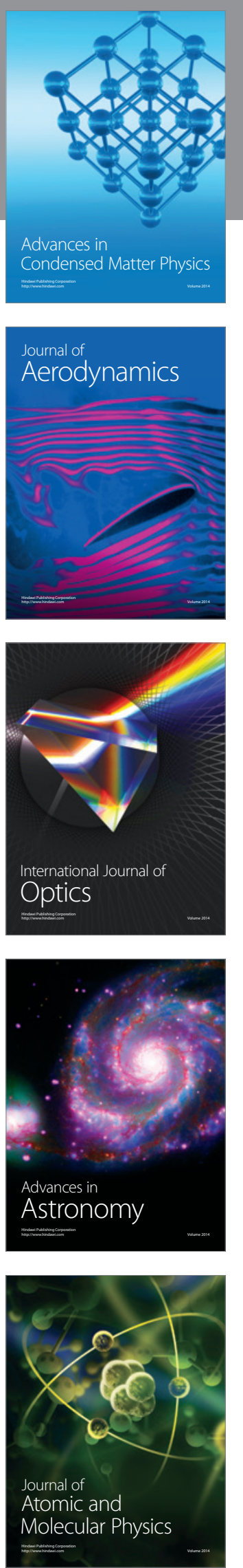